\title{
Anti-inflammatory Drugs for Cancer Prevention and Treatment: Emerging Options
}

\section{Reddanna Pallu*}

School of Life Sciences, University of Hyderabad, Hyderabad-500046, India

National Institute of Animal Biotechnology, Hyderabad-500046, India

Inflammation is a major component in the onset and progression of a variety of cancers. While chronic inflammation promotes carcinogenesis, cancers in turn will have inflammatory microenvironment. This is evident from a number of epidemiological studies showing reduced incidence of cancers in those on long term use of non-steroidal anti-inflammatory drugs (NSAIDs) [1] and the COX-2 selective anti-inflammatory drugs (COXIBs) [2]. Not only do the NSAIDs suppress the inflammatory microenvironment in tumors but also alleviate the cancer pain associated with chemotherapy [3] and in patients with advanced stages of cancer. These studies also led to unravel the molecular pathways linking inflammation and cancer, and the cancer related inflammation (CRI) has come to be recognized as the Seventh Hallmark of cancer [4]. Targeting inflammation for the prevention and treatment of cancers, either alone or in combination with the chemotherapeutic drugs is therefore gaining importance.

The inflammatory tumor microenvironment is known to contribute to proliferation and survival of malignant cells, angiogenesis, metastasis, subversion of adaptive immunity and diminish the response to hormones and chemotherapeutic agents. The accumulating evidences stemming from different lines of work clearly demonstrate the utility of anti-inflammatory drugs and the role of inflammation in the promotion of cancers [5]. Epidemiological data as well as the data from clinical trials have clearly demonstrated a decreased incidence of primary and recurrent cancers in those on long-term NSAID therapy. Decreased mortality was also reported in cancer patients undergoing combination therapy involving NSAIDs. In view of GI toxicity associated with the conventional NSAIDs, the selective COX-2 inhibitors (COXIBs) with reduced GI toxicity gained importance over NSAIDs in the early 2000s. Studies in humans indicate that COXIBs could effectively control inflammation and were considered good candidates for prevention and treatment of cancer [6,7]. However, very soon it became evident that long-term use of COXIBs increases the cardiac side effects and this resulted in the withdrawal of rofecoxib by Merck \&Co in Sept. 2004 and later a number of other COXIBs (Valdecoxib, Etoricoxib, and Parecoxib). The shunting of the substrate arachidonic acid towards the 5-LOX pathway, as a result of the inhibition of COX pathway, was shown to increase the production leukotrienes, which contribute to the side effects of COXIBs [8]. This realization formed the basis for the development of COX-2/5-LOX dual inhibitors (CLOXIBs) as a new series of anti-inflammatory drugs without gastric or cardiac side effects. Licofelone (ML-3000) is one such well characterized CLOXIB that possesses anti-inflammatory effects with reduced GI toxicities and no cardiac side effects [9].

With the increased realization and emphasis on the lifestyle changes in preventing/controlling effectively the metabolic syndromes including cancer, there is a worldwide shift towards the traditional/folk medicines, at least as a last resort for survival by the patients/or their wards. A number of natural compounds such as resveratrol, curcumin and chebulagic acid [10], which inhibits both cyclooxygenases and lipoxygenases, have anti-inflammatory and anti-cancer effects and could be a great promise for cancer prevention and treatment. However, there is need for more intensive studies on the validation of such natural systems of medicine in terms of efficacy and safety, besides making efforts for developing natural product based anti-inflammatory drugs through reverse engineering practices. These approaches not only could lead to the quick development of safe and effective antiinflammatory drugs but also make them affordable for public at large.

\section{References}

1. Schreinemachers DM, Everson RB (1994) Aspirin use and lung, colon, and breast cancer incidence in a prospective study. Epidemiology 5: 138-146.

2. Bardou M, Barkun AN, Ghosn J, Hudson M, Rahme E (2004) Effect of chronic intake of NSAIDs and cyclooxygenase 2-selective inhibitors on esophageal cancer incidence. Clin Gastroenterol Hepatol 2: 880-887.

3. Suraneni MV, Reddy GV, Reddanna P (2011) Horizons in Cancer Research Watanabe HS (Editor), Nova Science Publishers 47: 1-18.

4. Colotta F, Allavena P, Sica A, Garlanda C, Mantovani A (2009) Cancer-related inflammation, the seventh hallmark of cancer: links to genetic instability. Carcinogenesis 30: 1073-1081.

5. Agarwal S, Reddy GV, Reddanna P (2009) Eicosanoids in inflammation and cancer: the role of COX-2. Expert Rev Clin Immunol 5: 145-165.

6. Gupta RA, Dubois RN (2001) Colorectal cancer prevention and treatment by inhibition of cyclooxygenase-2. Nat Rev Cancer 1: 11-21.

7. Rayburn ER, Ezell SJ, Zhang R (2009) Anti-Inflammatory Agents for Cancer Therapy. Mol Cell Pharmacol 1: 29-43.

8. de Gaetano G, Donati MB, Cerletti C (2003) Prevention of thrombosis and vascular inflammation: benefits and limitations of selective or combined COX1, COX-2 and 5-LOX inhibitors. Trends Pharmacol Sci 24: 245-252.

9. Rao P, Knaus EE (2008) Evolution of nonsteroidal anti-inflammatory drugs (NSAIDs): cyclooxygenase (COX) inhibition and beyond. J Pharm Pharm Sci 11: $81 \mathrm{~s}-110 \mathrm{~s}$.

10. Reddy DB, Reddy TC, Jyotsna G, Sharan S, Priya N, et al. (2009) Chebulagic acid, a COX-LOX dual inhibitor isolated from the fruits of Terminalia chebula Retz., induces apoptosis in COLO-205 cell line. J Ethnopharmacol 124: 506512.

*Corresponding author: Reddanna Pallu, School of Life Sciences, University of Hyderabad, National Institute of Animal Biotechnology, Hyderabad-500046, India, Tel: 914-023-010-745; Fax: 914-023-010-745; E-mail: prsl@uohyd.ernet.in/ preddanna@niab.org.in

Received October 29, 2012; Accepted October 31, 2012; Published November 02, 2012

Citation: Reddanna P (2012) Anti-inflammatory Drugs for Cancer Prevention and Treatment: Emerging Options. J Cancer Sci Ther 4: xxvii-xxvii. doi:10.4172/19485956.1000e117

Copyright: (c) 2012 Reddanna P. This is an open-access article distributed unde the terms of the Creative Commons Attribution License, which permits unrestricted use, distribution, and reproduction in any medium, provided the original author and source are credited. 